\title{
Um estudo sobre alfabetização financeira com acadêmicos de administração em uma instituição de ensino superior no interior do Ceará
}

O objetivo deste estudo foi identificar qual o nível de alfabetização financeira dos acadêmicos do curso de administração da Faculdade Vale do Salgado no município de Icó, no Estado do Ceará, a partir do Termômetro de Alfabetização Financeira (POTRICH et al., 2016). Para tanto se fez necessário compreender o perfil sócio econômico dos discentes do curso de administração, bem como verificar se os discentes em sua vida cotidiana têm práticas de alfabetização financeira e distinguir quais os níveis de alfabetização financeira dos discentes, constituem-se como objetivos específicos. A pesquisa utilizou abordagem quantitativa de caráter exploratório e descritivo, onde foi realizado um estudo de caso na IES. Com aplicação do questionário: Termômetro de Alfabetização Financeira sendo os dados analisados através do software Statistical Package for the Social Sciences - SPSS. Conclui-se, para que aconteça o bem-estar financeiro é necessário compreender a importância da alfabetização financeira, que é um processo indispensável na formação social e intelectual. Com base no exposto, os acadêmicos apresentam um nível médio de conhecimento, porém, é imprescindível um desenvolvimento do conhecimento dentro da temática para ampliar e aperfeiçoar habilidades, sobretudo na administração das finanças pessoais, buscando planejamento para qualidade de vida e bem-estar financeiro.

\section{A study on financial literacy with business students at a higher education institution in the interior of Ceará}

\begin{abstract}
The objective of this study was to identify the level of financial literacy of the academic students of the administration course of the Faculdade Vale do Salgado, in the city of Icó, in the State of Ceará, from the Financial Literacy Thermometer (POTRICH et al., 2016). Therefore, it was necessary to understand the socio-economic profile of the students of the course of administration, as well as to verify if the students in their daily life have financial literacy practices and to distinguish the levels of financial literacy of the students, constituting the specific objectives of this study. The research used an exploratory and descriptive quantitative approach, carrying out a case study in IES. With application of the questionnaire: Financial Literacy Thermometer, the data was being analyzed through the software Statistical Package for the Social Sciences - SPSS. In order for financial well-being to happen, it has been concluded that it is necessary to understand the importance of financial literacy, an indispensable process in social and intellectual formation. Based on what was presented, the students present an average level of knowledge, however, it is essential to develop knowledge within the theme to expand and improve skills, especially in the management of personal finances, seeking planning for quality of life and well-being financial.
\end{abstract}

Keywords: Financial Literacy; Financial literacy thermometer; Administration students; Icó-Ceará

Topic: Finanças Empresariais

Reviewed anonymously in the process of blind peer.
Received: 19/11/2019

Approved: 20/03/2020
Romário Medeiros Cândido

Centro Universitário Vale do Salgado, Brasil

http://lattes.cnpq.br/4908000562895619

medeiros.candido25@gmail.com

Antoniel dos Santos Gomes Filho (it)

Centro Universitário Vale do Salgado, Brasil

http://lattes.cnpq.br/9563145614494252

http://orcid.org/0000-0003-2230-4315

antonielsantos@univs.edu.br

Otácio Pereira Gomes

Centro Universitário Vale do Salgado, Brasil http://lattes.cnpq.br/3296784496379104

http://orcid.org/0000-0003-3532-8412

otaciopereira@univs.edu.br
Maria Erilucia Cruz Macêdo

Centro Universitário Vale do Salgado, Brasil http://lattes.cnpq.br/4333538235010417

mariaerilucia@hotmail.com

\section{Ednael Macedo Felix (iD}

Universidade de Fortaleza, Brasil

http://lattes.cnpq.br/1231915953131426

http://orcid.org/0000-0002-4520-8257

edynaell@hotmail.com

Antonio Wilson dos Santos

Centro Universitário Vale do Salgado, Brasil

http://lattes.cnpq.br/9085815217693327

wilson@univs.edu.br

Referencing this:

CÂNDIDO, R. M.; GOMES FILHO, A. S.; GOMES, O. P.; MACÊDO, M. E. C.; FELIX, E. M.; SANTOS, A. W.. Um estudo sobre alfabetização financeira com acadêmicos de administração em uma instituição de ensino superior no interior do Ceará. Business Journal, v.2, n.1, p.1227, 2020. DOI: http://doi.org/10.6008/CBPC2674-6433.2019.001.0002 


\section{INTRODUÇÃO}

Com o cenário econômico e financeiro complexo que vem se agravando nas últimas décadas a alfabetização financeira surge como possível solução que precisa ser estudada e compreendida. Embora sua importância seja reconhecida, pesquisas desenvolvidas assinalam que uma grande parcela da população sofre com analfabetismo financeiro e que se faz necessário medidas para amenizar essa situação (LUSARDI et al., 2011; POTRICH et al., 2016; KUHL et al., 2016).

A conjuntura econômica mundial vem sofrendo oscilações de mercado ao longo de toda sua história, tais como desestímulo aos investimentos, estagnação da industrialização e aumento dos preços, tendo se agravado nos últimos anos com destaque para os países emergentes, no qual o Brasil se enquadra. Diante deste cenário, pode-se perceber que muitos brasileiros percebem a crise como algo já naturalizado por conta das questões sociais, visto que é sentida de forma direta pela população se tornando concreta. Conforme o SEBRAE (2013), a falta de organização financeira é um dos principais fatores que levam a uma instabilidade econômica pessoal, pois contribui para o elevado número de inadimplentes que só tendem a permanecer nesse ciclo de endividamento. Essa realidade atinge inclusive estudantes universitários.

A falta de organização financeira de estudantes universitários já foi alvo de estudos e investigações noutros pontos do país como mostra Ferreira et al. (2016), em sua pesquisa com estudantes de Uberlândia/MG, apontam que a maioria dos alunos cerca de $80 \%$, não tem dinheiro disponível e apresenta dificuldades com as finanças pessoais. Assinala ainda, que apenas $20 \%$, buscam informações sobre finanças pessoais levando a conclusão que muitos não têm conhecimento sobre educação financeira para controle dos seus próprios gastos e investimentos. Lacerda et al. (2016) destacam que estudantes de Campina Grande/PB, concordam em sua maioria ( $56 \%$ concordam plenamente e $35 \%$ concordam parcialmente), que o tema educação financeira é essencial para trabalhar com os universitários para que esses não se tornem endividados.

Nesse contexto está sendo estudado e discutido mundialmente o termo alfabetização financeira, pois com base em estudos desenvolvidos nos últimos anos foram identificados que esse conceito sintetiza toda essa problemática. A alfabetização financeira é fundamental para desenvolver estratégias na administração dos rendimentos, poupanças e investimentos, visto que, mediante o cenário atual com a falta de conhecimento, se faz necessário manter um controle sobre a renda que se têm e sobre as despesas. Observando e abordando de forma empírica os acadêmicos de administração da Faculdade Vale do Salgado, localizado na cidade e Icó/CE, percebe-se a dificuldade no planejamento e controle da renda, mediante as despesas. Diante dessa perspectiva, questiona-se: Qual o nível de alfabetização financeira dos acadêmicos do curso de administração de uma Instituição de Ensino Superior do município de Icó, no Estado do Ceará?.

O presente estudo teve como objetivo principal: Identificar qual o nível de alfabetização financeira dos acadêmicos do curso de administração da Faculdade Vale do Salgado no município de Icó, no Estado do Ceará, a partir do Termômetro de Alfabetização Financeira (POTRICH et al., 2016). Para tanto se fez necessário compreender o perfil sócio econômico dos discentes do curso de administração, bem como 
verificar se os discentes em sua vida cotidiana têm práticas de alfabetização financeira e distinguir quais os níveis de alfabetização financeira dos discentes do curso de administração, constituem-se como objetivos específicos.

A ampliação dos estudos em Alfabetização Financeira na Região Centro Sul do Ceará é de grande importância para a circulação do conhecimento nesta área, pois como foi possível perceber através das pesquisas nas bases de dados, que não há estudos publicados, na região citada. O conhecimento adquirido ao longo da pesquisa proporcionara um desenvolvimento em todas as particularidades que envolvem o tema na vida pessoal e profissional do pesquisador. Dessa forma, atribui um conjunto de habilidades, bem como técnicas para gestão dos rendimentos pessoais, poupanças e investimentos. A pesquisa pode ser utilizada para fomenta os conhecimentos já adquiridos ao longo da graduação no que tange o aperfeiçoamento das finanças pessoais e na aplicação para vida profissional. Vale ressaltar que a pesquisa cientifica dentro dessa área é de fundamental importância, onde contribuirá para o corpo docente e discente para novas pesquisas.

\section{REVISÃO TEÓRICA}

\section{Gestão Financeira}

Compreende-se que a gestão financeira está integrada à um conjunto de processos e ações administrativas. Segundo Assaf Neto et al. (2014), o processo de administração financeira é desenvolvido com base nas seguintes funções: Planejamento financeiro, Controle financeiro, Administração de ativos e administração de passivos. Já para Lacerda et al. (2016), o planejamento financeiro é, sobretudo, a forma mais racional de administrar a renda, buscando equilibrar um conjunto de fatores para se alcançar os objetivos estabelecidos.

Dessa forma, Alves et al. (2016) enfatizam que desde a efetivação do plano real ao longo dos últimos anos, vem ocorrendo estímulos para o consumo, com o acesso ao crédito que foi popularizado e ligado às mídias de comunicação, que apresentam ofertas diárias para comercialização de produtos, bens e serviços. Nisso, Silva et al. (2015) dizem que, os indivíduos com maior facilidade de endividamento consideram que os baixos salários é um fator para utilização do cheque especial e que o limite do cartão de crédito é considerado como renda.

Assim, de acordo com o Banco Central do Brasil (2017), a instabilidade econômica sofrida nos últimos anos, ocasionou uma desaceleração no crescimento do crédito disponível à pessoa física, voltando a mostrar sinais de recuperação apenas em 2017. Nesse contexto, Silva et al. (2015) destacam que com a falta de planejamento, os indivíduos comprometem boa parte de sua renda, tendo em vista o consumismo excessivo que possibilita uma inadimplência, levando à desonra seus acordos financeiros.

De acordo com Zemiacki et al. (2015), as mudanças no cenário econômico trouxeram também uma nova perspectiva para projeção futura do país, com base na necessidade de aprendizagem financeira. Para Cerbasi (2015), esse momento da economia é uma oportunidade para corrigir erros e refazer planos, pois, se faz necessário sair da ideia de crise e consequentemente, que tudo está negativo, sobretudo compreendendo 
que o país vive um momento de ajuste.

\section{Finanças Pessoais}

As finanças pessoais envolvem uma série de ações quanto ao gerenciamento dos rendimentos particulares. Segundo Lizote et al. (2016), as finanças pessoais são entendidas como a forma que envolve o manuseio do dinheiro, sendo esse próprio ou não, para ter acesso ao mercado, tendo o controle de como ganhar e gastar bem. Porém, Alves et al. (2016) afirmam que a população, em sua grande maioria, não apresenta a devida preparação para adequar o que se ganha com as despesas adquiridas, podendo se deixar levar pela inadimplência, tendo em vista o aumento desnorteado de crédito.

De acordo com Conto et al. (2015), no Brasil existe a necessidade de pesquisas mais aprofundadas na administração das finanças pessoais, visto que se faz necessário entender o comportamento das pessoas. Dessa forma, Alves et al. (2016) enfatizam que o endividamento é um fato contemporâneo que engloba todos os países, independente da classe social e formação acadêmica, transparecendo além da vida pessoal do indivíduo e prejudicando nas relações com a família, no trabalho e nos diversos vínculos sociais; além de ser um assunto que é possível desenvolver pesquisas em diversas áreas.

Assim, Souza et al. (2014), afirmam que a população brasileira, em sua grande maioria, não possui consciência financeira, preocupa-se apenas em suprir necessidades básicas. O conhecimento em finanças pessoais baseia-se em estudos de métodos fundamentais no desenvolvimento de habilidades para gestão dos recursos pessoais. A poupança, investimentos e planejamento, observando sempre a necessidade de curto e longo prazo, são fatores que contribuem para melhores resultados na vida financeira (FRANCISCHETTI et al., 2014).

Compreende-se que a atitude financeira está integrada à um conjunto de conhecimento e ações. Segundo Lacerda et al. (2016), a atitude financeira é à capacidade dos indivíduos usarem o conhecimento, as habilidades e a confiança que adquiriram, sobretudo, a consequência da forma mais racional de administrar a renda, buscando comportamento para equilibrar fatores na tentativa de se alcançar o bem-estar financeiro.

O comportamento financeiro baseia-se em métodos fundamentais no desenvolvimento de habilidades para gestão dos recursos pessoais. 0 estímulo para poupar, saber investir e planejar, são fatores que contribuem para melhores resultados na vida financeira (FRANCISCHETTI et al., 2014). O conhecimento em finanças pessoais é o processo pelo qual consumidores e investidores melhoram sua compreensão sobre produtos, conceitos e riscos financeiros. Com isso, conseguem desenvolver o conhecimento e consequentemente a confiança para tomar decisões financeiras responsáveis (FRANCISCHETTI et al., 2014).

\section{Alfabetização Financeira}

Por muito tempo o termo alfabetização foi caracterizado, de grosso modo, apenas como codificação e decodificação dos códigos que era considerado suficiente para identificar o sujeito como alfabetizado. Sendo assim, o processo de educar era exclusivamente responsável por ensinar aos alunos tais códigos. Atualmente entende-se que o conceito de alfabetização supera a restrição de codificar e decodificar, como 
classificação generalizada para esse processo (RIOS, 2015).

O processo de alfabetização ocorre quando o indivíduo se apropria do conhecimento, que vai além das escolas e instituições de ensino, pois necessita de uma aprendizagem significativa. $\mathrm{O}$ ambiente que o aluno está inserido, especialmente familiar, também influência expressivamente para alcançar essa alfabetização, que é de extrema importância (SILVA et al., 2014).

A Organization for Economic Co-operationand Development (OECD, 2013), conceitua a alfabetização financeira como uma combinação de algumas habilidades necessárias, sendo estas: consciência financeira; conhecimento financeiro; habilidade financeira; atitude financeira; e comportamento financeiro. São elementos necessários para os indivíduos tomarem as decisões financeiras, e, contudo, buscando obter resultados satisfatórios no desempenho econômico.

Potrich et al. (2016) apontam que, a alfabetização financeira apesar de ser constantemente relacionada com educação financeira e conhecimento financeiro têm suas diferenças, pois ela aborda conceitos que vão além dos apontados pelo ensino e pela noção que se tem sobre finanças. É necessário entender que existe uma diferença entre os termos: alfabetização financeira, educação financeira e conhecimento financeiro, onde, pode haver problemas se um destes termos for usado como sinônimo do outro (KUHL et al., 2016). Já para Silva et al. (2017) o simples conhecimento a respeito de finanças pessoais, não é o requisito exclusivo e suficiente para que as pessoas sejam consideradas alfabetizadas financeiramente.

Huston (2010) enfatiza que a alfabetização financeira se baseia na compreensão da educação desta aliada às informações sobre o financeiro pessoal, pois a parti desses conhecimentos e sua aplicação, que é possível alcançar as dimensões da alfabetização financeira. De acordo com Kuhl et al. (2016), se faz necessário compreender o real significado do termo alfabetização financeira dando sua importância na aplicação dentro do contexto diário de cada indivíduo.

Deste modo, Potrich et al. (2015) afirmam ainda que, enquanto a educação financeira possibilita o entendimento acerca do processo que facilite as decisões referentes à administração das finanças pessoais, a alfabetização financeira é a competência que engloba todos esses conhecimentos e habilidades, tendo a capacidade de realizar sua utilização. Lusardi et al. (2011) enfatizam que é importante analisar como os indivíduos são financeiramente alfabetizados, tendo em vista que na realidade, ou seja, na prática é complexo entender e explorar como de fato as pessoas interpreta e processa as informações financeiras, para tomar decisões com base nesse conhecimento.

Nisso, Kuhl et al. (2016) destacam que com base na literatura verifica-se o conceito de que a alfabetização financeira é considerada multidimensional, de forma que não é possível abranger todas as suas dimensões, levando em consideração apenas um construto. Portanto, não há um consenso no meio acadêmico sobre as mais viáveis ferramentas para mensuração da alfabetização supracitada.

Os indivíduos com baixos níveis de alfabetização financeira são considerados mesmo que inconscientemente, mais predispostos à praticarem falhas financeiras, sobretudo apresentando maior dificuldade em lidar com variações econômicas imprevistas. Esses baixos níveis podem ainda ocasionar a falta 
de investimento nos mercados financeiros e consequentemente elevar o número de empréstimo, sendo adquirida a parte de fontes informais, tendo elevadas taxas de juros (KLAPPER et al., 2013).

A importância da alfabetização financeira é mundialmente reconhecida como elemento de estabilidade e desenvolvimento econômico e financeiro, que foi retratado na aprovação dos Princípios de alto Nível sobe Estratégias Nacionais para a Alfabetização Financeira da OECD, endossado para o encontro do G20 (OECD, 2013).

\section{METODOLOGIA}

\section{Tipo de Pesquisa}

A pesquisa possui abordagem quantitativa e de caráter exploratório, onde foi realizado um estudo de caso na Faculdade Vale do Salgado, em específico, no curso de Bacharelado em Administração. De acordo com Marconi et al. (2017), a abordagem quantitativa é mais apropriada nas ciências sociais, uma vez que é empregado questionário para apurar atitudes e responsabilidades dos entrevistados. Para Gil (2017), o estudo de caso consiste numa profunda e exaustiva pesquisa, permitindo uma ampla e detalhada visão sobre o objeto estudado, independentemente de ser um ou mais objetos. Conforme Gil (2016), a pesquisa exploratória consiste em desenvolver uma maior familiaridade com o problema, pois busca esclarecer e modificar conceitos e consequentemente facilite estudos posteriores.

\section{Área de estudo}

A pesquisa foi aplicada na Faculdade Vale do Salgado (FVS), localizada na Av. Monsenhor Frota, 609 - Centro, Icó/Ce. A população representa os discentes do curso de administração da faculdade Vale do Salgado, sendo estes com idade igual ou superior a 18 anos. Consistiu como critérios de inclusão: acadêmicos devidamente matriculados na IES. Como critérios de exclusão, serão os alunos que estiverem, no período da coleta de dados, em algum tipo de afastamento, tal como licença maternidade, doença ou acidente, bem como não estarem dispostos a participar da pesquisa. Foi aplicado um questionário estruturado.

\section{População e amostra}

Para levantar informações necessárias à população a ser amostrada foi composta por 157 estudantes do Curso de Graduação em Administração da Faculdade Vale do Salgado (FVS), no município de Icó, obedecendo aos seguintes critérios de inserção: discentes do curso, regularmente matriculados e que aceitarem participar da pesquisa por meio da assinatura do Termo de Consentimento Pós Esclarecido (TCPE). O tamanho da amostra foi definido a partir do cálculo amostral para obtenção do tamanho da amostra seguida a fórmula adotada por Martins (2005), que é expressa na seguinte forma:

$$
n=\frac{\mathrm{Z}^{2} \cdot \mathrm{p} \cdot \mathrm{q} \cdot \mathrm{N}}{d^{2} \cdot(\mathrm{N}-1)+\mathrm{z}^{2} \cdot \mathrm{p} \cdot \mathrm{q}}
$$


$p=$ estimativa da proporção da característica pesquisada no universo;

Admitindo-se a população de discentes ( $N=157)$, um erro de estimação de $(\mathrm{d}=5 \%)$; abscissa do normal padrão $(z=95 \%)$ ao nível de confiança de $95 \%$ e $(p=q=0,5)$ na hipótese de se admitir o maior tamanho da amostra, porquanto não se conhece as proporções estudadas, obteve-se um tamanho de amostra (n) igual a 112 questionários (MARTINS, 2005). Contudo, por conta de informações inconsistentes, foram excluídos, dois questionários, totalizando uma amostra final de 110.

\section{Tipo de análise}

Para caracterizar o grau de atitude, comportamento e conhecimento financeiro dos alunos da Faculdade Vale do Salgado (FVS), Icó, Ceará, foi empregado à análise fatorial. Conforme Fávero et al. (2009) o método de análise fatorial consiste na tentativa de se determinar as relações quantitativas entre as variáveis, aferindo seus padrões de movimento, de modo a associar, àquelas com um padrão semelhante, o efeito de um fator causal subjacente e específico a estas variáveis. O modelo matemático, conforme Fávero et al. (2009) da análise fatorial poderá ser representado por:

$$
\begin{gathered}
Z_{11=} a_{11} F_{1}+a_{12} F_{2+\ldots+} a_{1 m} F_{m+} d_{1} u_{1} \\
Z_{21=} a_{21} F_{1+} a_{22} F_{2+\ldots}+a_{2 m} F_{m+} d_{2} u_{2} \\
Z_{n}=a_{n 1} F_{1+} a_{n 2} F_{2+\ldots+} a_{n m} F_{m+} d_{n} u_{n}
\end{gathered}
$$

De forma simplificada, tem-se:

$$
Z_{j=} \sum a_{j i} F_{i+} d_{j} u_{j}(j=1,2, \ldots, n) ;(i=1,2, \ldots, m)
$$

Tal que:

$$
Z_{j=\text { j-ésima variável padronizada; }}
$$

$a_{j i}$ = é o coeficiente de saturação referente ao i-ésimo fator comum da j-ésima variável;

$F_{i}=$ é o i-ésimo fator comum;

$d_{j}=$ é o coeficiente de saturação referente ao j-ésimo fator específico da j-ésima variável;

$u_{j}=$ é o j-ésimo fator específico da j-ésima variável.

De acordo com a análise fatorial, cada fator é constituído por uma combinação linear das variáveis originais inseridas no estudo. Dentro da análise fatorial, através da verificação da matriz de correlação, devem ser apresentados os coeficientes de correlação de Pearson para cada par de variáveis. A relação entre as variáveis deve ser confirmada a partir do nível de significância dos coeficientes estimados $(p-v a l u e<0,05)$.

De acordo com Hair Junior et al. (2005), a análise iniciará a partir do exame da matriz de correlações para verificação da existência de valores significativos que justifiquem a utilização da técnica. Deve ser considerada também a normalidade dos dados para o uso da técnica (utilizando o Teorema do Limite Central, que caso haja um grande número de variáveis aleatórias independentes e identicamente distribuídas, então a distribuição tenderá para uma distribuição normal, à medida que o número dessas variáveis aumente indefinidamente). Ainda segundo os autores, se a visualização da matriz de correlações não mostrar um número substancial de valores maiores que 0,30 , haverá fortes indícios que a análise fatorial não será 
adequada.

Para verificar a adequabilidade dos dados a utilização da análise fatorial, foi utilizado o índice KaiserMayer-Olkin (KMO), o teste de esfericidade de Bartlett (BTS) e a Matriz Anti-Imagem. O índice Kaiser-MayerOlkin (KMO), que varia de 0 a 1 , serve para comparar as magnitudes dos coeficientes de correlações observados com as magnitudes dos coeficientes de correlações parciais. Portanto, o KMO trata-se de uma medida de homogeneidade das variáveis, que compara as correlações parciais observadas entre as variáveis, conforme a equação (2) a seguir:

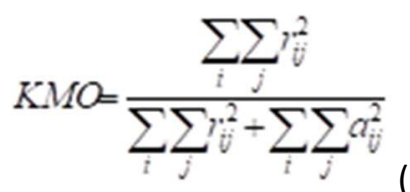

$r_{i j}$ é o coeficiente de correlação observado entre as variáveis $i$ e $j$

$a_{\ddot{v}}$ é o coeficiente de correlação observado entre as mesmas variáveis, que é, simultaneamente, uma estimativa das correlações entre os fatores. Os ${ }^{a} i j$ deverão estar próximos de zero, pelo fato de os fatores serem ortogonais entre A estatística do KMO (Kaiser-Mayer-Olkin) é a seguinte: quanto menor o valor do respectivo teste, menor a relação entre as variáveis e os fatores, podendo o índice variar entre 0 e 1 , sendo que o índice menor que $(0,5)$ caracteriza-se como inaceitável o uso dessa técnica, quanto mais o índice for próximo de 1, maior será a adequabilidade dos dados a utilização da técnica.

O teste Bartlett de esfericidade testará a hipótese nula de que a matriz de correlação é uma matriz identidade, se por ventura isso ocorrer inviabilizará a utilização da análise fatorial. Caso a matriz de correlações seja uma matriz identidade, significa que as inter-relações entre as variáveis são iguais a zero e, portanto, a análise fatorial não deverá ser utilizada.

A matriz anti-imagem também mostra a partir da matriz de correlações a adequabilidade dos dados à análise fatorial e apresenta os valores negativos das correlações parciais. Na sua diagonal principal são apresentados os valores de MSA (Measure of Sampling Adequacy) ou a Medida de Adequação da Amostra, para cada variável, ou seja, quanto maiores esses valores, melhor será a utilização da análise fatorial e, caso contrário, talvez seja necessário excluí-la da análise (HAIR JUNIOR et al., 2005).

A extração dos fatores iniciais foi através da Análise dos Componentes Principais (ACP) que mostrou uma combinação linear das variáveis observadas, de maneira a maximizar a variância total explicada. A escolha do número de fatores se deu por meio do critério da raiz latente (critério de Kaiser) em que se escolheu o número de fatores a reter, em função dos valores próprios acima de 1 (eigenvalues). Com a finalidade de minimizar a dificuldade de interpretação dos fatores, utilizou-se o método de rotação ortogonal (mantendo-se a independência entre eles) Varimax, que minimizou o número de variáveis com altas cargas em diferentes fatores permitindo a associação de uma variável a um único fator, mantendo a ortogonalidade entre eles.

\section{Cálculo do Índice grau de Atitude Financeira, Comportamento Financeiro e Conhecimento Financeiro}


A análise fatorial permitiu criar um índice que pode mensurar o grau de atitude, comportamento e conhecimento financeiro dos alunos da FVS, no município de Icó, Ceará. O índice de Atitude Financeira, Comportamento Financeiro e Conhecimento Financeiro pode ser representado da seguinte equação matemática:

$$
I N D I C E_{i}=\sum_{j=1}^{p}\left[\frac{\lambda_{j}}{\sum \lambda_{j}}\right] F_{i j} *
$$

Em que: ÍNDICE = Índice de Atitude, Comportamento ou Conhecimento Financeiro do i-ésimo entrevistado $j=$ é a j-ésima raiz característica; $p=$ é o número de fatores extraídos na análise;

$\sum \lambda_{j}=$ é o somatório das raízes características referentes aos $p$ fatores extraídos; A participação relativa do fator $j$ na explicação da variância total captada pelos $p$ fatores extraídos e indicada por $\frac{\hat{\lambda} j}{\sum \lambda j}$.

Para tornar todos os valores dos escores fatoriais $F^{*}$ superiores ou iguais a zero, todos eles foram colocados no primeiro quadrante (LEMOS, 2001), antes da construção do ÍNDICE , utilizando-se a expressão algébrica:

$$
F_{j i}^{*}=\frac{F_{j i}-F_{j}^{\min }}{F_{j}^{\max }-F_{j}^{\min }}
$$

Em que:

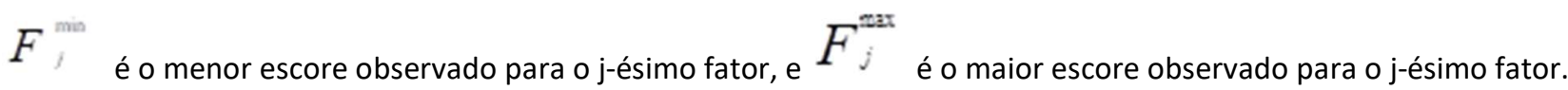
A coleta de dados foi desenvolvida por meio do formulário no Google Forms, realizada no mês de abril de 2018, nos dias 11 e 12. A análise dos dados ocorreu através da tabulação dos dados, com uso do software Statistical Package for the Social Sciences - SPSS

\section{RESULTADOS E DISCUSSÃO}

\section{Análise do perfil dos estudantes}

A tabela 1, abaixo constata-se que $53,6 \%$ dos entrevistados são do gênero masculino e $46,4 \%$, são do gênero feminino. A maioria dos respondentes é jovem que têm idade entre 20 a 25 anos correspondendo a $58,2 \%$, seguido dos que possuem idade inferior a 20 anos 33,6\%. Que em sua grande maioria já trabalhou ou trabalha $78,2 \%$, mas que atualmente apenas $59,1 \%$ possuem renda fixa.

Tabela 1: Perfil socioeconômico.

\begin{tabular}{|l|l|l|l|}
\hline Gênero: & Frequência & Porcentagem \\
\hline \multirow{3}{*}{} & Masculino & 59 & 53,6 \\
\cline { 2 - 4 } & Feminino & 51 & 46,4 \\
\cline { 2 - 4 } & Total & 110 & 100,0 \\
\hline
\end{tabular}




\begin{tabular}{|c|c|c|}
\hline Faixa etária: & Frequência & Porcentagem \\
\hline \begin{tabular}{l|l} 
& Menos de 20 anos
\end{tabular} & 37 & 33,6 \\
\hline De 20 a 25 anos & 64 & 58,2 \\
\hline De 26 a 35 anos & 9 & 8,2 \\
\hline \begin{tabular}{ll}
\cline { 2 - 2 } & Total \\
\end{tabular} & 110 & 100,0 \\
\hline Você já trabalhou ou trabalha: & Frequência & Porcentagem \\
\hline \begin{tabular}{l|l|} 
Sim \\
\cline { 2 - 2 }
\end{tabular} & 86 & 78,2 \\
\hline Não & 24 & 21,8 \\
\hline \begin{tabular}{ll}
\cline { 2 - 2 } & Total \\
\end{tabular} & 110 & 100,0 \\
\hline Renda mensal pessoal: & Frequência & Porcentagem \\
\hline \begin{tabular}{l|l} 
& Até $1 \mathrm{~S}$ \\
\cline { 2 - 2 }
\end{tabular} & 48 & 43,6 \\
\hline De 1 a $3 \mathrm{~S}$ & 17 & 15,5 \\
\hline Nenhuma renda & 45 & 40,9 \\
\hline Total & 110 & 100,0 \\
\hline
\end{tabular}

\section{Análise da confiabilidade dos constructos}

Em função do objetivo da presente pesquisa, os respondentes foram questionados referentes aos constructos 'Atitude Financeira', 'Comportamento Financeiro' e 'Conhecimento Financeiro', o primeiro foi constituído por meio de 10 perguntas, e o segundo, por meio de 27 perguntas, e o terceiro constructo sendo representado por 13 perguntas. O questionário foi estruturado para que se pudesse captar com mais detalhes os aspectos que envolvem a concepção de finanças pessoais. A Tabela 2, abaixo, contém as médias e desvios padrões das perguntas referentes aos constructos. A consistência internamente dos constructos foi avaliada por meio do cálculo Alpha de Cronbach. Os valores variam entre 0 e 1 , medidas mais altas sugerem maior coerência entre os indicadores (HAIR JUNIOR et al., 2005).

Analisando a Tabela 2, quando analisado o constructo Atitude Financeira, notou-se que as perguntas que se mostravam razoavelmente importante, com médias iguais ou acima de 2 foram: P2 $(2,00)$ e P9 $(2,02)$. Já para o constructo Comportamento Financeiro as perguntas foram: P22 $(2,03)$ e P32 $(2,05)$. Para o constructo Conhecimento Financeiro se tem as perguntas: P38 (2,31), P39 $(2,09)$, P40 $(2,48)$, P41 $(2,33)$, P42 $(2,08)$, P43 (2,82), P44 $(2,08)$, P46 $(2,82)$ e P48 $(3,11)$.

$\mathrm{Na}$ outra extremidade, as perguntas com média inferior a 2 quando analisado o constructo Atitude Financeira foram: P3 (1,94), P4 $(1,58)$, P5 $(1,94)$, P6 $(1,78)$, P7 $(1,41)$, P8 $(1,36)$ e P10 $(1,89)$. Já para o constructo Comportamento Financeiro as perguntas foram: P11 (1,70), P12 (1,23), P13 $(1,58)$, P14 $(1,62)$, P15 (1,52), P16 (1,55), P17 (1,72), P18 (1,54), P19 (1,41), P20 (1,96), P21 (1,96), P23 (1,19), P24 (1,57), P25 (1,79), P26 (1,48), P27 (1,40), P28 (1,75), P29 (1,99), P30 (1,75), P31 $(1,88)$, P33 $(1,93)$, P34 $(1,18)$, P35 $(1,99)$, P36 $(1,78)$ e P37 $(1,79)$. Para o constructo Conhecimento Financeiro tem-se as perguntas: P45 $(1,75)$, P47 $(1,49)$, P49 $(1,55)$ e P50 (1,35). A partir desses resultados, subentende-se que, os estudantes estão conscientes quanto ao P38 (2,31), P39 (2,09), P40 (2,48), P41 (2,33), P42 (2,08), P43 (2,82), P44 $(2,08)$, P46 $(2,82)$ e P48 $(3,11)$.

Entretanto, apesar de ter percebido uma consciência dos estudantes por meio das algumas perguntas individuais, nota-se, que dos constructos estruturado inicialmente, apenas Atitude Financeira apresentou coerência interna não significativa. Os demais apresentaram Alpha de Cronbach em níveis considerados ótimos (acima de 0,60). Para o 1ำ Constructo Atitude Financeira, não possui um constructor considerado confiável, mas nada que comprometesse a análise fatorial (AF). Para o 20 e 3 o Constructo se 
apresentaram adequados quanto à confiabilidade dos seus componentes.

Tabela 2: Estatística descritiva dos Indicadores.

\begin{tabular}{|c|c|c|c|c|}
\hline PERGUNTAS & Média & Desvio padrão & Constructo Proposto & Alpha de Crobanch \\
\hline P2 & 2,00 & ,383 & \multirow{9}{*}{ 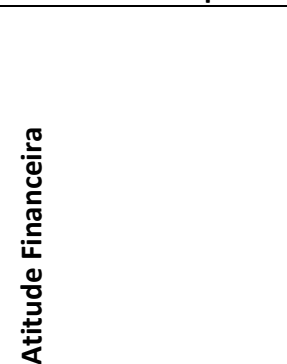 } & \multirow[t]{9}{*}{0,305} \\
\hline P3 & 1,94 & ,625 & & \\
\hline P4 & 1,58 & ,839 & & \\
\hline P5 & 1,94 & 870 & & \\
\hline P6 & 1,78 & ,794 & & \\
\hline P7 & 1,41 & ,746 & & \\
\hline P8 & 1,36 & ,700 & & \\
\hline P9 & 2,02 & ,469 & & \\
\hline P10 & 1,89 & ,902 & & \\
\hline P11 & 1,70 & ,698 & \multirow{27}{*}{ 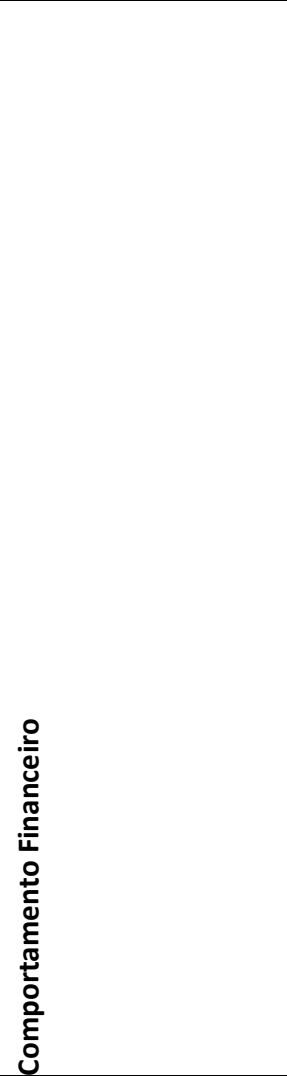 } & \multirow[t]{27}{*}{0,822} \\
\hline P12 & 1,23 & ,585 & & \\
\hline $\mathrm{P} 13$ & 1,58 & ,759 & & \\
\hline P14 & 1,62 & ,690 & & \\
\hline P15 & 1,52 & ,739 & & \\
\hline P16 & 1,55 & ,819 & & \\
\hline P17 & 1,72 & ,900 & & \\
\hline P18 & 1,54 & ,673 & & \\
\hline P19 & 1,41 & ,770 & & \\
\hline $\mathrm{P} 20$ & 1,96 & ,789 & & \\
\hline P21 & 1,96 & ,605 & & \\
\hline $\mathrm{P} 22$ & 2,03 & ,582 & & \\
\hline P23 & 1,19 & ,550 & & \\
\hline P24 & 1,57 & ,772 & & \\
\hline P25 & 1,79 & 791 & & \\
\hline P26 & 1,48 & ,751 & & \\
\hline P27 & 1,40 & ,706 & & \\
\hline P28 & 1,75 & 710 & & \\
\hline P29 & 1,99 & ,642 & & \\
\hline P30 & 1,75 & , 578 & & \\
\hline P31 & 1,88 & 787 & & \\
\hline P32 & 2,05 & ,548 & & \\
\hline P33 & 1,93 & ,483 & & \\
\hline P34 & 1,18 & ,492 & & \\
\hline P35 & 1,99 & ,723 & & \\
\hline P36 & 1,78 & ,722 & & \\
\hline P37 & 1,79 & 836 & & \\
\hline P38 & 2,31 & 1,029 & \multirow{13}{*}{ 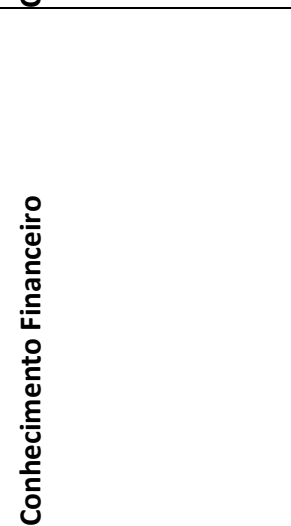 } & \multirow[t]{13}{*}{0,737} \\
\hline P39 & 2,09 & ,973 & & \\
\hline $\mathrm{P} 40$ & 2,48 & 1,179 & & \\
\hline P41 & 2,33 & 1,126 & & \\
\hline P42 & 2,08 & 1,015 & & \\
\hline P43 & 2,82 & 911 & & \\
\hline P44 & 2,08 & 1,158 & & \\
\hline P45 & 1,75 & 818 & & \\
\hline P46 & 2,82 & ,930 & & \\
\hline P47 & 1,49 & $\begin{array}{l}787 \\
\end{array}$ & & \\
\hline P48 & 3,11 & ,580 & & \\
\hline P49 & 1,55 & ,773 & & \\
\hline P50 & 1,35 & ,685 & & \\
\hline
\end{tabular}

Em termos gerais, a análise fatorial trabalhou com o problema de analisar a estrutura das correlações entre um grande número de variáveis (itens de testes, respostas de questionários e etc.), definindo um conjunto de dimensões latentes comuns, chamadas de fatores (HAIR JUNIOR et al., 2005, citado por SILVA, 2016).

\section{Resultados da análise fatorial (AF)}


As próximas etapas da pesquisa foram exploradas os conjuntos de respostas das perguntas de cada constructo da Tabela 3, abaixo, tendo como foco elaborar uma nova estrutura latente que explicasse os níveis dos estudantes quanto à atitude, comportamento e conhecimento financeiro tendo em vista, que estrutura inicial não apresentou estatística em níveis, no ponto de vista teórico, aceitáveis. Portanto, no primeiro momento, avaliou-se o teste de 'esferidade de Bartlett's' e o teste 'Kaiser-Meyer-Olkin (KMO)', e derivando por fim o modelo fatorial.

Tabela 3: Teste de KMO (Kaiser Mayer Olkin) e BTS (Teste de Esfericidade de Bartlett).

\begin{tabular}{|c|c|c|c|}
\hline \multirow[t]{4}{*}{ MODELO GERAL } & \multicolumn{2}{|l|}{ KMO. } & ,560 \\
\hline & \multirow[t]{3}{*}{ Teste de esfericidade de Bartlett } & Chi-quadrado aprox. & 1826,492 \\
\hline & & Df & 1176 \\
\hline & & Sig. & 0,000 \\
\hline \multirow[t]{4}{*}{ ATITUDE FINANCEIRA } & \multicolumn{2}{|l|}{ KMO. } & ,527 \\
\hline & \multirow[t]{3}{*}{ Teste de esfericidade de Bartlett } & Chi-quadrado aprox. & 425,961 \\
\hline & & $\mathrm{Df}$ & 235 \\
\hline & & Sig. & 0,0098 \\
\hline \multirow[t]{4}{*}{ COMPORTAMENTO FINANCEIRO } & \multicolumn{2}{|l|}{ KMO. } & 697 \\
\hline & \multirow[t]{3}{*}{ Teste de esfericidade de Bartlett } & Chi-quadrado aprox. & 767,988 \\
\hline & & Df & 351 \\
\hline & & Sig. & 0,000 \\
\hline \multirow[t]{4}{*}{ CONHECIMENTO FINANCEIRO } & \multicolumn{2}{|l|}{ KMO. } & ,743 \\
\hline & \multirow[t]{3}{*}{ Teste de esfericidade de Bartlett } & Chi-quadrado aprox. & 260,416 \\
\hline & & $\mathrm{Df}$ & 78 \\
\hline & & Sig. & 0,000 \\
\hline
\end{tabular}

O teste de Bartlett's testa a presença de correlações entre os indicadores (perguntas). O teste KaiserMeyer-Olkin (KMO) fornece uma medida para quantificar o grau de intercorrelações entre os indicadores de forma geral e em cada constructor. Assim, inicialmente, com o intuito de verificar a coesão das informações gerais, foi calculado o teste Kaiser-Mayer-Olkin (KMO), observou-se, pela Tabela 3 considerando-se distribuição normal dos dados, que o KMO-geral revelou valor de 0,560 , portanto, indicando que os dados são consistentes, ou seja, é um índice razoável e aceito para análise fatorial. O Teste de Esfericidade de Bartlett indicou significante a $1 \%$ (sig.=0,000), rejeitando a hipótese nula $\left(\mathrm{H}_{0}\right)$ de que a matriz de correlação é uma matriz-identidade. Conclui-se, portanto, que os dados amostrais de forma geral são adequados para uso da análise fatorial.

Quando analisados de forma isolada cada constructor, percebe-se que em relação à Atitude Financeira o teste verificou um p-valor (significância) de 0,000 e um KMO de 0,527, sendo considerado consistente, mas medíocre, ou seja, mesmo assim indicando a presença de correlações entre as variáveis. Para o constructor Comportamento Financeiro, o KMO revelou valor de 0,697, portanto, indicando que os dados são consistentes, ou seja, é um índice razoável e aceito para análise fatorial, já o Teste de Esfericidade de Bartlett indicou significante a $1 \%($ sig. $=0,000)$.

Por fim, quando analisado para o constructor Conhecimento Financeiro, o teste verificou um p-valor (significância) de 0,000 e um KMO de 743, sendo considerada consistente, ou seja, mesmo assim indicando a presença de correlações entre as variáveis. Portanto, de acordo com os resultados dos testes de adequabilidade da análise fatorial, foi possível utilizá-la para a criação dos índices propostos. 
Tabela 4: Valores das raízes características e percentual de variância total explicada pela análise fatorial.

\begin{tabular}{|l|l|}
\hline COSNTRUTOR & Variância acumulada (\%) \\
\hline ATITUDE FINANCEIRA & 57,60 \\
\hline COMPORTAMENTO FINANCEIRO & 62,92 \\
\hline CONHECIMENTO FINANCEIRO & 55,78 \\
\hline
\end{tabular}

Fonte: Resultados da Pesquisa (2018)

A Tabela 4 acima mostra a variância acumulada explicada pelo modelo de forma geral, assim como por cada constructor. Dessa forma pode-se perceber que tanto de uma forma geral como quando avaliado cada constructor de forma individual, a variância acumulada apresenta-se bem elevada, sendo maior o grau de explicabilidade do modelo no constructor Comportamento Financeiro.

Tabela 5: As variáveis que mais explicam cada constructor ${ }^{1}$

\begin{tabular}{|l|l|}
\hline MODELO & VARIÁVEIS \\
\hline ATITUDE FINANCEIRA & $\mathrm{p} 3 ; \mathrm{p} 6 ; \mathrm{p} 7 ; \mathrm{p} 8$ \\
\hline COMPORTAMENTO FINANCEIRO & $\mathrm{p} 17 ; \mathrm{p} 20 ; \mathrm{p} 24 ; \mathrm{p} 25 ; \mathrm{p} 26 ; \mathrm{p} 26$ \\
\hline CONHECIMENTO FINANCEIRO & $\mathrm{p} 42 ; \mathrm{p} 44 ; \mathrm{p} 46 ; \mathrm{p} 47 ; \mathrm{p} 48 ; \mathrm{p} 49 ; \mathrm{p} 50$ \\
\hline
\end{tabular}

Frente a estes resultados, que a priori já demonstraram possuir níveis de qualidades adequadas à sua utilização como instrumento para avaliar o índice, foi possível observar quais variáveis representavam melhor explicabilidade dentro de cada constructo. Assim, pode-se perceber que no constructo Atitude financeira as variáveis que mais contribuíram para explicar foram: P3(poupar é impossível para nossa família); P6(planejamento de gastos familiar); P7(gastar dinheiro em coisas importantes); e P8(gastos presentes que afetam o futuro). Já para o constructo Comportamento Financeiro destacam-se as variáveis: P17(objetivos determinados ao gerenciar o dinheiro); P20(guarda parte da renda mensal); P24(dinheiro suficiente para pagar as despesas); P25(organização de registros financeiros); e P26(comprar por impulso). Por fim, para o constructor Conhecimento financeiro as variáveis foram: P42(retorno de ativo); P44(distribuição de investimento); P46(taxa de juros sobre empréstimos); P47(utilização do melhor desconto); P48(dividir dinheiro igualmente); P49(taxa de risco); e P50(quando a inflação aumenta).

\section{Análise dos índices}

De acordo com a Tabela 6 abaixo, percebe-se que foram obtidas três classes para classificação do nível de alfabetização dos acadêmicos de administração. Os grupos foram caracterizados como muito baixo; baixo; médio; alto; e muito alto. Esta escolha de 3 grupos tomou como base o trabalho de Cunha et al. (2008) que desenvolveu estudos semelhantes sobre classificação e agrupamentos de índices, mediante melhor estruturação das variáveis e sua quantidade.

Para o grupo classificado como muito baixo, que varia do intervalo de 0 a 0,20 , não existe nenhum aluno nesse nível, ou seja, de forma generalizada, os acadêmicos que participaram da pesquisa encontramse em um nível aceitável de acordo com as respostas dos pesquisados, do grupo dos que se consideram nível baixo onde varia de 0,21 a 0,35 existem 23 alunos com frequência relativa de 20,91\%. Já do grupo que apresenta nível médio onde varia de 0,37 a 0,62 existem 68 alunos com frequência relativa de $61,82 \%$ e os

\footnotetext{
${ }^{1}$ Valores acima 0,3 
que estão no nível alto, esses representam um percentual de 17,27\% dos entrevistados.

Tabela 6: Agrupamento segundo os grupos do índice atitude financeiro dos acadêmicos de administração.

\begin{tabular}{|l|l|l|l|}
\hline & $\mathbf{f i}$ & fri (\%) & Fi (\%) \\
\hline BAIXO & 23 & 20,91 & 26 \\
\hline MÉDIO & 68 & 61,82 & 94 \\
\hline ALTO & 19 & 17,27 & 113 \\
\hline TOTAL & 110 & 100,00 & \\
\hline
\end{tabular}

De acordo com a Tabela 6 acima, observa-se que dos estudantes entrevistados 20,91\% destes encontram com baixo nível de atitude financeira, já em sua maioria 61,82\% se encontram com atividade financeira média e apenas $17,27 \%$ destes apresentam uma atitude financeira alta.

Tabela 7: Agrupamento segundo os grupos do índice comportamento financeiro dos acadêmicos de administração.

\begin{tabular}{|l|l|l|l|}
\hline & $\mathbf{f i}$ & fri (\%) & FI \\
\hline BAIXO & 23 & 20,91 & 18 \\
\hline MÉDIO & 78 & 70,91 & 101 \\
\hline ALTO & 9 & 8,18 & 110 \\
\hline TOTAL & 110 & 100 & \\
\hline
\end{tabular}

Na tabela 7 acima, quando analisado o índice em relação ao comportamento financeiro observa-se que dos estudantes entrevistados $20,91 \%$ destes encontram com baixo nível de comportamento financeira, já em sua maioria $70,91 \%$ se encontram com comportamento financeira média e apenas $8,18 \%$ destes apresentam um comportamento financeiro alto.

Tabela 8: Agrupamento segundo os grupos dos índices de conhecimento financeiro dos acadêmicos de administração.

\begin{tabular}{|l|l|l|l|}
\hline & $\mathbf{f i}$ & $\mathbf{f r i}(\%)$ & $\mathbf{F I}$ \\
\hline BAIXO & 18 & 16,36 & 18 \\
\hline MÉDIO & 77 & 70,00 & 95 \\
\hline ALTO & 11 & 10,00 & 106 \\
\hline MUITO ALTO & 4 & 3,64 & 110 \\
\hline TOTAL & 110 & 100,00 & \\
\hline
\end{tabular}

De acordo com a Tabela 8 acima, observa-se que dos estudantes entrevistados $16,36 \%$ destes encontram com baixo nível de conhecimento financeira, já em sua maioria 70,00\% se encontram com conhecimento financeiro médio. Já $10,00 \%$ destes apresentam um conhecimento financeiro alto, e observase que $3,64 \%$ aponta um nível de conhecimento financeiro muito alto.

\section{CONCLUSÕES}

O processo de aprendizagem está diretamente ligado ao desenvolvimento do homem, pois é capaz de aprender em todas as etapas de sua vida, interagindo com a sociedade na construção do conhecimento. Nisso, para que ocorra o bem-estar financeiro é necessário compreender a importância da alfabetização financeira que é um processo indispensável na formação social e intelectual. Entender a parte financeira se faz necessário para conseguir administrar a renda. Dessa forma, alfabetização financeira é essencial, pois envolve um conjunto de habilidades que se desenvolve através do conhecimento, de forma consciente, buscando o comportamento adequado para as tomadas de decisões financeiras (OECD, 2013).

Considerando a importância deste estudo, que teve por objetivo identificar qual o nível de 
alfabetização financeira dos acadêmicos do curso de administração, levando em consideração o Termômetro de Alfabetização Financeira. Os resultados apontam que dentro das três variáveis, atitude financeira, comportamento financeiro e conhecimento financeiro, o nível geral dos acadêmicos é médio. Em relação às variáveis, observou-se que o conhecimento financeiro apresentou exclusivamente um nível muito alto, que não foi alcançado pelas demais variáveis.

Com base no exposto, percebe-se a necessidade de trabalhar com a temática alfabetização financeira dentro do ambiente acadêmico, pois é imprescindível para o desenvolvimento do conhecimento dentro da temática. Assim, ampliar e aperfeiçoar habilidades, sobretudo na administração das finanças pessoais, buscando planejamento para qualidade de vida e bem-estar financeiro. Sugere-se, para pesquisas futuras, expandir a amostra trabalhando com outros cursos da faculdade, como também em outras IES, tendo em vista a possibilidade de investigação e comparação de dados determinantes, sobretudo no que é tocante a alfabetização financeira.

\section{REFERÊNCIAS}

ASSAF NETO, A.; LIMA, F. G.. Curso de Administração Financeira. 3 ed. São Paulo: Atlas, 2014.

ALVES, H. H. S.; FLORISSI, S.. O endividamento do servidor público no Brasil: o caso da Universidade Federal do Rio Grande do Sul. Dissertação (Mestrado em Economia) Universidade Federal do Rio Grande do Sul, Porto Alegre, 2016.

BCB. Banco Central do Brasil. Relatório de Estabilidade Financeira: panorama do sistema financeiro. BCB, 2017.

CERBASI, G.. Autor de best-seller diz que momento de crise é propício para investimentos. 2015.

CONTO, S. M.; FALEIRO, S. N.; FUHR, I. J.; KRONBAUER, K. A. O comportamento de alunos do ensino médio do vale do Taquari em relação às finanças pessoais. Revista REEN. Florianópolis, v.8, n.2, 2015.

FERREIRA, M. T. L.; CAMPANHOLO, T. C. P.. O nível de educação financeira e finanças pessoais dos alunos da Universidade Federal de Uberlândia/MG. Belo Horizonte, 2016.

FRANCISCHETTI, C. E.; CAMARGO, L. S. G.; SANTOS, N. C.. Qualidade de vida, sustentabilidade e educação financeira. Revista de finanças e contabilidade da UNIMEP, Piracicaba, v.1, n.1, 2014.

FÁVERO, L. P. L.; BELFIORE, P. P.; SILVA, F. L.; CHAN, B. L. Analise de Dados: Modelagem Multivariada para Tomada de Decisões. Rio de Janeiro: Campus, 2009.

GIL, A. C.. Como elaborar projetos de pesquisa. 5 ed. São Paulo: Atlas 2017.

GIL, A. C.. Métodos e técnicas de pesquisa social. 6 ed. São Paulo: Atlas 2016.

HAIR JÚNIOR, J. F.; ANDERSON, R. E.; TATHAM, R. L.; BLACK, W. C.. Análise multivariada de dados. Porto Alegre:
Bookman, 2005.

HUSTON, S. J.. Measuring financial literacy. The Journal of Consumer Affairs, v.44, n.2, p.296-316, 2010. DOI: http://dx.doi.org/10.1111/i.1745-6606.2010.01170.x

KLAPPER, L.; LUSARDI, A.; PANOS, G. A.. Financial literacy and its consequences: Evidence from Russia during the financial crisis. Journal of Banking \& Finance, v.37, p.39043923, 2013.

KUHL, M. R.; VALTER, T.; GUSMÃO, I. B.. Alfabetização financeira: evidências e percepções em uma cooperativa de crédito. Sociedade, contabilidade e gestão, Rio de Janeiro, v.11, n.2, 2016.

LUSARDI, A.; MITCHELL, O. S.. Financial literacy and retirement planning in the United States. Journal of Pension Economics and Finance, v.10, n.4, p.509-525, 2011.

LIZOTE, S. A.; LANA, J.; VERDINELLE, M. A.; SIMAS, J.. Finanças pessoais: um estudo envolvendo os alunos de ciências contábeis de uma instituição de ensino superior. Revista da UNIFEB, Brusque, v.1, n.19, 2016.

LACERDA, L. I. S.; NEVES, A. I. P.. Estudo sobre finanças pessoais: educação financeira dos universitários de Campina Grande/PB. Monografia (Bacharelado em administração) Universidade Estadual da Paraíba, João Pessoa, 2016.

LEMOS, J. J. S.. Indicadores de degradação no Nordeste Subsumido e Semiárido. In: CONGRESSO BRASILEIRO DE ECONOMIA E SOCIOLOGIA RURAL, 34. Anais. Brasília: SOBER, 2001. p.1-10.

MARTINS, M. E. G.. Introdução as Probabilidades e Estatísticas. Lisboa: Sociedade Portuguesa de Estatística, 2005.

OECD. Organization for Economic Co-Operation and Development. Financial literacy and inclusion: Results of OECD/INFE survey across countries and by gender. Paris, 
OECD Centre, 2013.

POTRICH, A. C. G.; VIEIRA, K. M.; KIRCH, G.. Você é alfabetizado financeiramente? Descubra no termômetro de alfabetização financeira. Revista base, Administração e contabilidade da UNISINOS, São Leopoldo, v.13, n.2, p.153170, 2016.

POTRICH, A. C. G.; VIEIRA, K. M.; KIRCH, G.. Determinantes da alfabetização financeira: análise da influência de variáveis socioeconômicas e demográficas. Revista Cont. Fin., São Paulo, v.26, n.69, p.362-377, 2015.

RIOS, A. A. S.. prática pedagógica no processo de alfabetização. In: CONGRESSO NACIONAL DE EDUCAÇÃO, 12. Anais. Curitiba, 2015. p.2836-2848.

SEBRAE. Educação financeira para pessoa física: serviço de apoio às micro e pequenas empresas. Salvador: SEBRAE, 2013.

SILVA, F. C.; SILVA, J. G.. 'Devo não nego...' uma análise da gestão financeira pessoal dos consumidores de Ituiutaba/MG. In: SINGEP, 4. Anais. São Paulo, 2015.

SILVA, G. O.; SILVA, A. C. M.; VIEIRA, P. R. C.; DESIDERATI, M. C.; NEVES, M. B. E.. Alfabetização financeira versus educação financeira: um estudo do comportamento de variáveis socioeconômicas e demográficas. Revista de gestão, finanças e contabilidade, Salvador, v.7, n.3, p.279-298, 2017.

SILVA, M. G.; SILVA, F. P.. Processo de alfabetização. Monografia (Bacharelado em pedagogia EAD) - Universidade Estadual da Paraíba Catolé do Rocha, 2014.

SILVA, R.. Finanças pessoais e educação financeira: o perfil dos servidores públicos de um munícipio do centro-oeste brasileiro. Dissertação (Mestrado) - Fundação Instituto Capixaba de Pesquisas em Contabilidade, Economia e Finanças, Vitória, 2016.

SOUZA, J. C.; GUIMARÃES, M. L. F.. Manual de finanças pessoais: maneiras de gerenciamento das finanças pessoais para a formação de patrimônio. Monografia (Bacharelado em Ciências Contábeis) - Universidade do Extremo Sul Catarinense, Criciúma, 2014.

ZEMIACKI, J.; MACÊDO, G. R.. Educação financeira: uma metodologia de pesquisa amostral para aferição de indicadores em fundos de pensão. Dissertação (Mestrado) Universidade Federal do Rio Grande do Sul. Porto Alegre, 2015.

A CBPC - Companhia Brasileira de Produção Científica (CNPJ: 11.221.422/0001-03) detém os direitos materiais desta publicação. Os direitos referem-se à publicação do trabalho em qualquer parte do mundo, incluindo os direitos às renovações, expansões e disseminações da contribuição, bem como outros direitos subsidiários. Todos os trabalhos publicados eletronicamente poderão posteriormente ser publicados em coletâneas impressas sob coordenação da Cognitionis Publishing, da Companhia Brasileira de Produção Científica e seus parceiros autorizados. Os (as) autores (as) preservam os direitos autorais, mas não têm permissão para a publicação da contribuição em outro meio, impresso ou digital, em português ou em tradução. 